\title{
Hearing Characterization in Oculoauriculovertebral Spectrum: A Prospective Study with 10 Patients
}

\author{
Thayse Bienert Goetze, ${ }^{1}$ Pricila Sleifer, ${ }^{2}$ Rafael Fabiano Machado Rosa, ${ }^{1,3,4}$ \\ Alessandra Pawelec da Silva, ${ }^{1}$ Carla Graziadio, ${ }^{3}$ and Paulo Ricardo Gazzola Zen ${ }^{1,3,4 *}$ \\ ${ }^{1}$ Graduate Program in Pathology, Universidade Federal de Ciências da Saúde de Porto Alegre (UFCSPA), Porto Alegre, RS, Brazil \\ ${ }^{2}$ Speech Language Pathology, Universidade Federal do Rio Grande do Sul (UFRGS), Porto Alegre, RS, Brazil \\ ${ }^{3}$ Clinical Genetics, UFCSPA and Complexo Hospitalar Santa Casa de Porto Alegre (CHSCPA), Porto Alegre, RS, Brazil \\ ${ }^{4}$ Graduate Program in Biosciences, Universidade Federal de Ciências da Saúde de Porto Alegre (UFCSPA), Porto Alegre, RS, Brazil
}

Manuscript Received: 31 March 2015; Manuscript Accepted: 23 September 2016

Oculoauriculovertebral spectrum (OAVS), also known as Goldenhar syndrome, is considered a condition associated to failing of embryogenesis involving the first and second branchial arches, leading to structural abnormalities arising from it. The aim of this study is to verify the hearing features presented by patients with OAVS and provide additional information that may contribute to improvement of speech therapy. The sample consisted of 10 individuals diagnosed with OAVS and cared for by the Clinical Genetics Service. All patients underwent objective assessment of auditory function through tonal and vocal audiometry. This evaluation was completed using TOAE and BERA. The patient's age ranged from 1 year and 9 months to 27 years and 4 months. At physical examination it was found that 10 had microtia, 7 preauricular tags, 6 low-set ears, 6 ear canal atresia, and 2 preauricular pits. Among the patients, five presented with abnormal hearing. Three patients had conductive hearing loss ranging from mild to moderate, and two patients had sensorineural hearing loss from mild to profound. Three patients had hearing loss in both ears. Speech-language disorders are common in children with OAVS. Thus, the referral to the audiologist and speech pathologist is indicated as soon as possible. Early recognition and detailed understanding of aspects related to the etiology, clinical features, and outcome of patients with OAVS are essential for their proper management. () 2016 Wiley Periodicals, Inc.

Key words: Oculoauriculovertebral spectrum; Goldenhar syndrome; hearing sciences; audiology; speech; language

\section{INTRODUCTION}

Oculoauriculovertebral spectrum (OAVS) [OMIM 164210], also known as hemifacial microsomia and Goldenhar syndrome, is considered a rare condition which has been etiologically associated to different causes, which includes environmental factors that can result in a blastogenesis disruption. OAVS usually involves facial
How to Cite this Article:

Goetze TB, Sleifer P, Rosa RFM, da Silva AP, Graziadio C, Zen PRG. 2017. Hearing characterization in oculoauriculovertebral spectrum: A prospective study with 10 patients.

Am J Med Genet Part A 173A:309-314.

asymmetry and mainly affects the right side of the face [Rollnick et al., 1987; Cohen et al., 1989]. It has an estimated prevalence of one case in 5,600-26,550 live births, affecting more males than females at a ratio of about 3:2 [Grabb, 1965; Poswillo, 1973; Melnick, 1980; Cohen et al., 1989].

The features verified in patients with OAVS are characterized by a wide spectrum that ranges from mild to severe unilateral cranial, face, and neck congenital anomalies. Involvement of spine, heart, and kidney is also common [Cohen et al., 1989].

Anomalies associated with OAVS have been observed in children of mothers exposed to several teratogenic agents as thalidomide, primidone, retinoic acid, anticoagulants and salicylates, ethyl alcohol, folate antagonists, anticonvulsants, vasoactive drugs, and smoking [Kleinsasser and Schlothane, 1964; Cohen et al., 1989; Gorlin et al., 2001; Werler et al., 2004]. Other factors that have been related to OAVS include vaginal bleeding in 2 nd trimester, which suggests abnormalities in the placenta and are likely to have a vascular nature [Werler et al., 2004]; twin pregnancies, in which anastomosis of vessels of the placenta can be observed both in

Conflicts of interest: None.

${ }^{*}$ Correspondence to:

Paulo Ricardo Gazzola Zen, Clinical Genetics-UFCSPA/CHSCPA, Rua Sarmento Leite, 245 / 403, CEP: 90050-170 Porto Alegre, RS, Brazil.

E-mail: paulozen@ufcspa.edu.br

Article first published online in Wiley Online Library

(wileyonlinelibrary.com): 24 October 2016

DOI 10.1002/ajmg.a.38017 
monozygotic as in dizygotic twins; hypoxia due to high altitude [Salvado et al., 2003]; intrauterine compression secondary to oligohydramnios [Cohen et al., 1989; Salvado et al., 2003] and hypertension [Salvado et al., 2003].

Cytogenetic abnormalities have been described in individuals with the phenotype of OAVS. Several chromosomal abnormalities have been detected and include deletion of the long arm of chromosome 5, trisomy 18, duplication of the long arm of chromosome 7, and supernumerary chromosome $\operatorname{der}(22) \mathrm{t}(11 ; 22)$ (Emanuel syndrome) [Cohen et al., 1989; Rosa et al., 2010]. Patients presenting features of OAVS and mutations in the goosecoid, TCOF1 and GLI2 genes have been described. However, they usually have a more symmetric involvement than that seen in patients with OAVS [Rahimov et al., 2006].

Ear malformations in patients with OAVS can range from complete aplasia to deformities in the external, middle and inner ear which may result in hearing loss. The ear canal may be completely absent, resulting in deafness (unilateral) in approximately $40 \%$ of cases. It is known that exposure to sound stimuli is an essential factor for the child to develop the appropriate language and maturation of the central auditory system. This aspect emphasizes the importance of early detection and intervention of hearing disorders [Vinay et al., 2009].

Currently, it is known that any type of hearing loss can compromise language, learning, cognitive development, and social inclusion of children [Mondain et al., 2005]. Thus, a better understanding of abnormalities associated with OAVS will enable an earlier diagnosis and better planning and treatment approach.

\section{METHODS}

The sample consisted of patients with OAVS originated from the Clinical Genetics Service of Universidade Federal de Ciências da Saúde de Porto Alegre (UFCSPA)/Complexo Hospitalar Santa Casa de Porto Alegre (CHSCPA). Using the Hospital database, 23 patients were initially identified. Later, an active search was conducted by telephone or letter contact. Of 23 patients initially selected, it was possible to contact 10 of them. All patients and their parents agreed to participate in the study. During the first contact, a protocol for the identification and the scheduled time of execution of speech therapy interventions were performed. All patients were re-evaluated to confirm the diagnosis of OAVS by medical geneticists. This followed the criteria suggested by Strömland et al. [2007]. Thus, we considered patients with OAVS those with clinical alterations in at least two of the following areas: (i) oro-craniofacial; (ii) ocular; (iii) auricular; and (iv) vertebral.

This is an observational, cross-sectional study, and all patients or guardians signed an informed consent form. The study was approved by the Ethics Committee of the institution. All patients had previously performed high-resolution GTGbanding karyotype in peripheral blood; fluorescent in situ hybridization (FISH) for $22 \mathrm{q} 11$ and $5 \mathrm{p}$ microdeletions, and search for chromosomal instability for Fanconi anemia. All presented normal results.

At first interview, it was asked information about auditory function, patient history, aspects of child development, and information about school performance. Abnormalities described in organs or systems, and results of ophthalmological, otorhinolaryngological, cardiological, and radiological evaluations were also registered. Anthropometric measurements of patients, such as weight, height, and head circumference, were verified. All patients underwent a speech-language study with the use of standard protocols, to assess auditory function and school performance for literate students (through the Academic Performance Test [APT]).

Audiological tests performed consisted of auditory brainstem audiometry or infant audiometry, speech audiometry, tympanometry, acoustic reflexes, and otoacoustic emissions. All tests were conducted in the Audiology Clinic of Hospital de Clínicas de Porto Alegre (HCPA). Evaluations were performed with the AC40 clinical audiometer and the AD229 diagnostic audiometer (Interacoustis ${ }^{\circledR}$ ) and TDH39 audiometric earphones (TDH Telephonics $\left.{ }^{\mathbb{B}}\right)$ in sound booths. The pure tone audiometry at frequencies of $250,500,1,000,2,000,3,000,4,000,6,000$, and $8,000 \mathrm{~Hz}$ was performed in most patients. Bone conduction frequencies of 500 , $1,000,2,000,3,000$, and $4,000 \mathrm{~Hz}$ were tested. The descending method was used for stimulus presentation. The analysis of the results was performed by calculating the average of three frequencies: 500, 1,000, and 2,000 dB. For the classification of hearing loss, we selected the scale of Davis and Silverman [1970].

The speech audiometry was performed by investigating speech recognition threshold (SRT). For this evaluation, trisyllabic words were presented to the patient in audible intensity, $40 \mathrm{~dB}$ above the tone average of airway. This intensity was reduced to below the threshold. The patient was instructed to repeat the heard words. It was considered a proper SRT when the patient correctly repeat at least $50 \%$ of them.

We also carried out the research of speech recognition index (SRI). The SRI was obtained using the presentation of a list of 25 monosyllabic words in intensity audible to the patient, $40 \mathrm{~dB}$ above the tone average of airway. This intensity was fixed during the whole test. It was demonstrated to the patient that he should repeat the heard word. If the patient responded $92-100 \%$, the assay was considered unchanged; but when he answered values less than $88 \%$, he underwent to more 25 two-syllable words and it was registered the percentage of correct reproduction. When the patient was unable to perform the SRT and SRI, due to problems as inability to repeat words (usually three syllables) or to perform simple orders, threshold of detection of voice (TDV) was used.

\section{RESULTS}

The final sample consisted of 10 subjects, five males, with age ranged from 1 year and 9 months to 27 years and 4 months, with a median of 4.5 years. Their birth, family, and clinical features can be seen in Table I. In only one pregnancy was reported twin pregnancy. One patient had no information about pregnancy, because the child was adopted (Table I).

Three patients presented a family history of hearing loss in individuals before 50 years of age. Regarding to auditory function, five patients complained of hearing loss in the right ear and three in both ears. Among these patients the greatest commitment was on the left. 


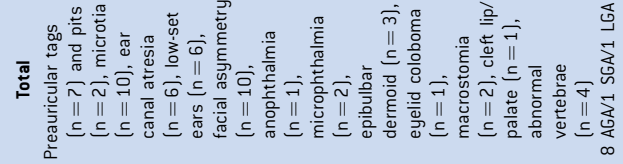

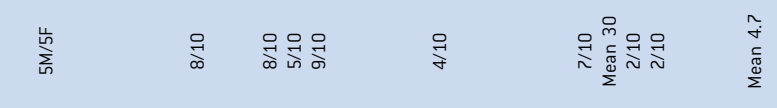

पू

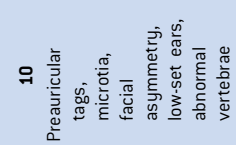

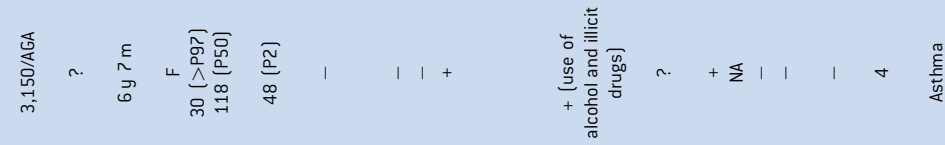

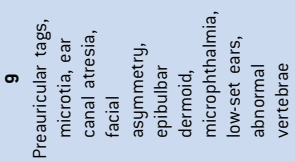

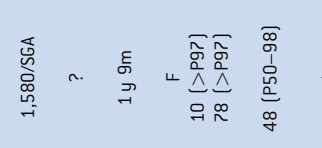

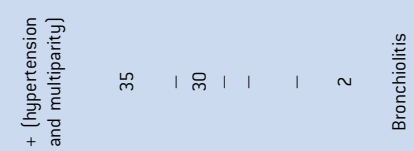

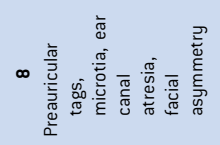

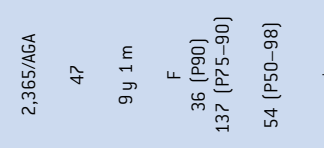

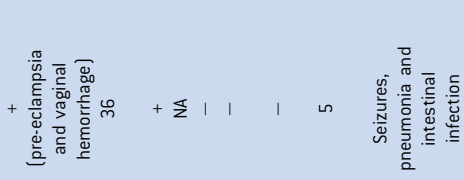

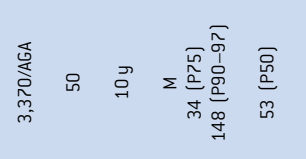

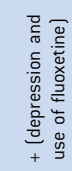

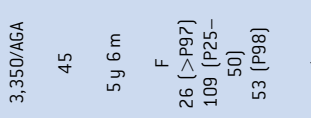

$\infty 121,1-\frac{2}{2}$

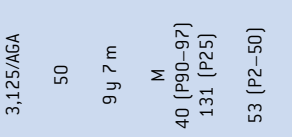

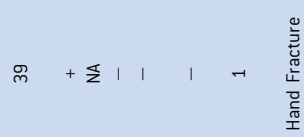

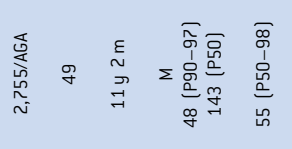

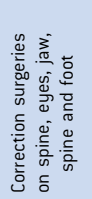

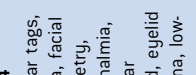

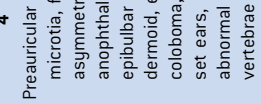

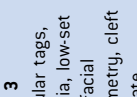

0
$m$

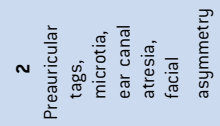

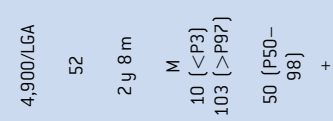

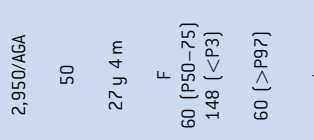

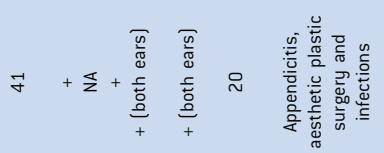

产

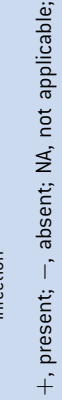

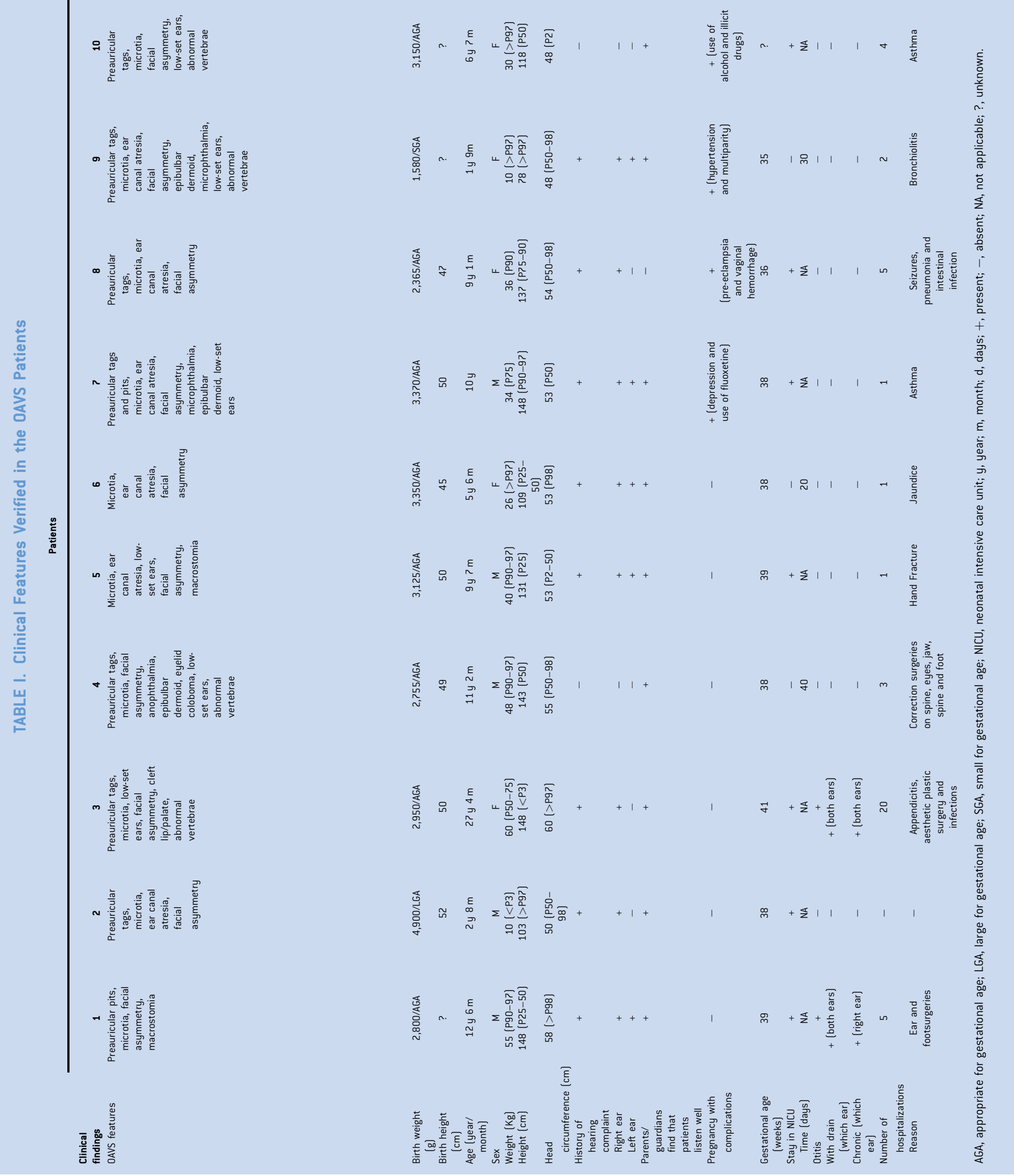




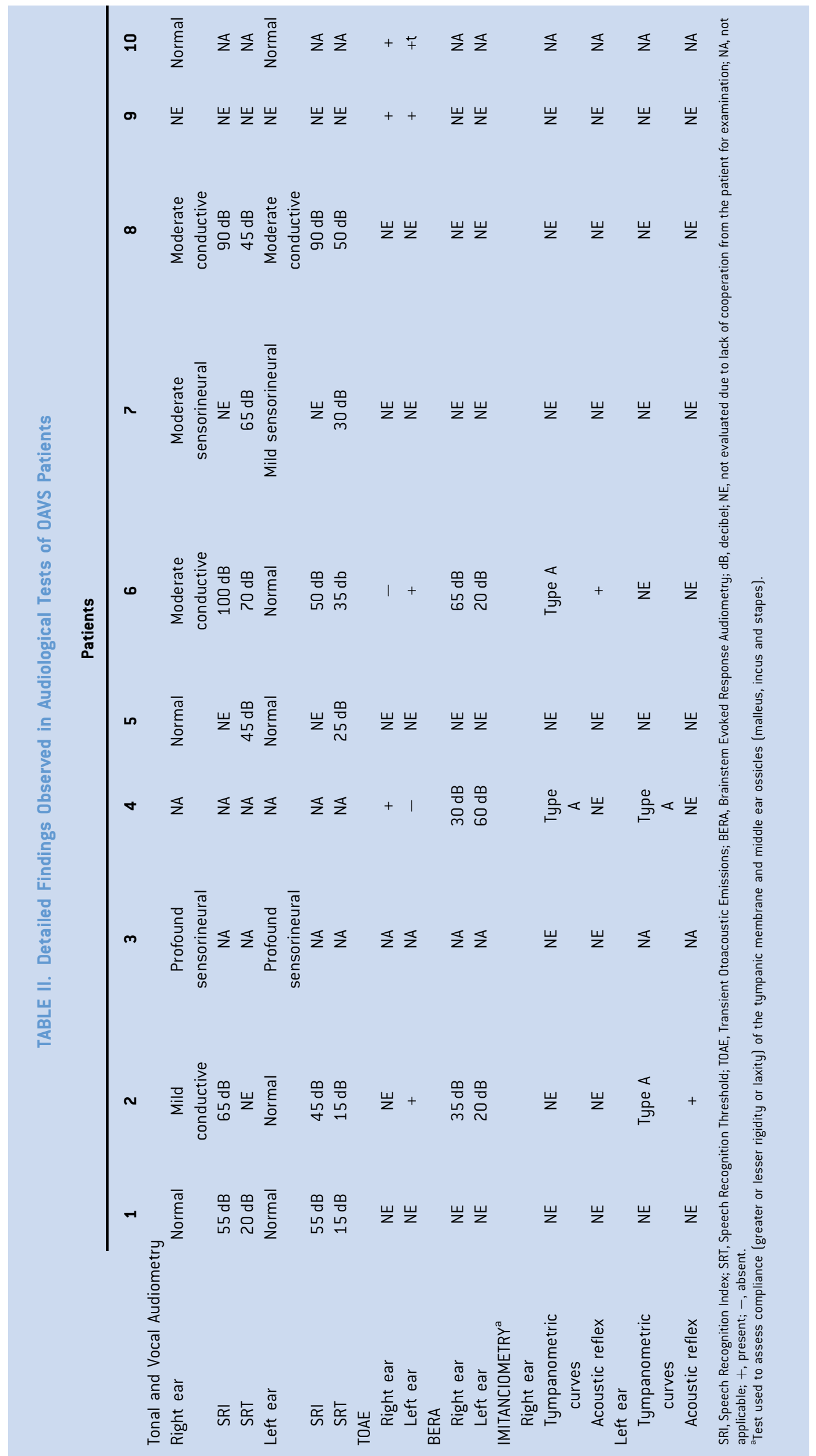


On physical examination, we verified that 10 patients had microtia, 7 preauricular tags, 6 low-set ears, 6 ear canal atresia, and 2 preauricular pits (Table I). As for neurological development, three had neuropsychomotor delay, and five language delay. The latter had, at the time of evaluation, changes in speech. Regarding schooling, five attend traditional school, four studying in a special school. Besides, five reported school failure or learning disabilities and, additionally, six had difficulty in concentration and attention. Five patients had visual difficulties.

APT could be performed in three children. They had a performance of $100 \%$, doing everything that was asked, in minimal time and with $100 \%$ of accuracy. The performance in basic skills of reading, writing, and arithmetic was considered satisfactory.

The findings of the audiological tests can be seen in Table II. Some patients did not undergo all evaluations due to lack of cooperation. From all, five presented an abnormal hearing. Three patients had conductive hearing loss ranging from mild to moderate, and two had sensorineural hearing loss from mild to profound. It is noteworthy that three patients had bilateral hearing loss. Two patients showed similar hearing thresholds due to excessive difficulty in performing the examination. At the time of the evaluation, two patients were evaluated using the Assessment Individual Sound Amplification Device (AISAD).

\section{DISCUSSION}

OAVS is characterized by a classic triad of ocular, auricular, and vertebral abnormalities [Rollnick et al., 1987; Cohen et al., 1989]. Ear abnormalities may involve middle, internal, and especially external ear [Rosa et al., 2011]. Microtia is the most common finding. Unilateral microtia is about six times more frequent than bilateral. Microtia is also more common in men than in women, being predominantly found in the right ear [Van Lierde et al., 2004]. In the present study, we observed that nine patients had microtia, with patients of both gender equally affected. In addition, several patients presented other ear findings, as preauricular tags, low-set ears, preauricular pits, and ear canal atresia. All these findings have been described within the spectrum of ear abnormalities described in OAVS.

Ear malformations are close related to hearing losses [Cohen et al., 1995]. However, it is noteworthy that are few studies evaluating the presence of hearing loss among patients with OAVS [Cohen et al., 1995; Brosco et al., 2004; Van Lierde et al., 2004; Engiz et al., 2007; Strömland et al., 2007]. Moreover, they did not usually detail aspects related to audiology symptoms or investigation. Thus, there is a lack of studies in literature reporting detailed aspects related to hearing of patients with OAVS. The findings verified in our sample draw attention to this aspect and the need for more auditory evaluation of these patients since hearing loss, both conductive as sensorineural, seems to be common.

The language development depends largely on hearing. Thus, a reduction or absence of hearing drastically reduces the ability to develop speech and language [Van Lierde et al., 2004]. Cohen et al. [1995] described that $92 \%$ of 24 patients with OAVS of their sample had some degree of hearing loss, which contribute to poor expressive and receptive language scores. From total, $37 \%$ and $32 \%$ of patients scored more than two standard deviations below the mean of the normal population in these domains, respectively. In our study, five children had language delay. These observations are consistent with those described by Brosco et al. [2004]. Any type of hearing loss can compromise language, learning, cognitive development, and social inclusion of children. These aspects reinforce the need for referral OAVS patients to the audiologist as soon as possible.

The effects of unilateral hearing loss are smaller than those caused by bilateral losses; however, they may also cause problems. In the presence of environmental noise, children with unilateral hearing loss may present greater difficulties than normal listeners to understand speech, even when the ear is positioned toward the sound source [Brosco et al., 2004]. Furthermore, it is thought that even with mild hearing losses, intelligible speech, and reading skills may be delayed or impaired [Yule and Rutter, 1987].

Early recognition and detailed understanding of aspects related to the clinical features and outcome of patients with OAVS are essential for their proper management. Usually, it is carried out in a multidisciplinary way due to the wide spectrum of clinical findings observed in OAVS. Therefore, several specialties, as Pediatrics, Medical Genetics, Otorhinolaryngology, Ophthalmology, Neurology, Psychiatry, Pediatric Surgery, and Speech Therapy, are frequently involved.

Thus, there is obvious need for further studies to investigate speech pathology associated with OAVS. This will delineate more clearly what abnormalities are associated with this syndrome and may indicate what type of radiological and laboratory investigation are the most appropriate. As a result, this will allow the early identification and intervention of these changes.

\section{REFERENCES}

Brosco KC, Zorzetto NL, Costa AR. 2004. Audiology profile in patients with Goldenhar's syndrome. Rev Bras Otorrinolaringol 70:645-649.

Cohen MM, Rollinck BR, Kaye CI. 1989. Oculoauriculovertebral spectrum: An updated critique. Cleft Palate J 26:276-286.

Cohen MS, Samango-Sprouse CA, Stern HJ, Custer DA, Vaught DR, Saal HM, Tifft CJ, Rosenbaum KN. 1995. Neurodevelopmental profile of infants and toddlers with oculo-auriculo-vertebral spectrum and the correlation of prognosis with physical findings. Am J Med Genet 60:535-540.

Davis H, Silverman SR. 1970. Auditory Test Hearing Aids. In: Davis H, Silverman SR, editors. Hearing and Deafness. Holt: Rinehart and Winston.

Engiz O, Balci S, Unsal M, Ozer S, Oguz KK, Aktas D. 2007. 31 cases with oculoauriculovertebral dysplasia (Goldenhar Syndrome): Clinical, neuroradiologic, audiologic, and cytogenetic findings. Genet Couns $18: 277-288$

Gorlin JJ, Cohen MM, Hennekam RC. 2001. Syndromes of the head and neck. London: Oxford University Press. pp 790-797.

Grabb WC. 1965. The first and second branchial arch syndrome. Plast Reconstr Surg 36:485-508.

Kleinsasser O, Schlothane R. 1964. Ear malformation in the framework of thalidomide embryopathy (Based on a survey of 70 infants bornduring 1959-1962). Z Laryngol Rhinol Otol 43:344-367. 
Melnick M. 1980. The etiology of external ear malformation and its relation to abnormalities of the middle ear, inner ear and other organ systems. Birth Defects Orig Artic Ser 16:303-331.

Mondain M, Blanchet C, Venail F, Vieu A. 2005. Classification et traitement des surdités de l'enfant. Oto-rhino-laryngologie (traité) 20: 190C-120C.

Poswillo D. 1973. The pathogenesis of the first and second branchial arch syndrome. Oral Surg Oral Med Oral Pathol 35:302-328.

Rahimov F, Ribeiro LA, de Miranda E, Richieri-Costa A, Murray JC. 2006. GLI2 mutations in four Brazilian patients: How wide is the phenotypic spectrum? Am J Med Genet Part A 140A:2571-2576.

Rollnick BR, Kaye CI, Nagatoshi K, Hauck W, Martin AO. 1987. Oculoauriculovertebral dysplasia and variants: Phenotypic characteristic of 294 patients. Am J Med Genet 26:361-375.

Rosa RFM, Pfeil JN, Zen PRG, Rosa RCM, Graziadio C, Paskulin AG. 2010. Phenotypical variability in supernumerary chromosome der$(22) t(11 ; 22)$ syndrome (Emanuel syndrome). Rev Paul Pediatr 28:367-371.

Rosa RFM, Silva AP, Goetze TB, Bier BA, Almeida ST, Paskulin GA, Zen PRG. 2011. Ear abnormalities in patients with oculo-auriculo-vertebral spectrum (Goldenhar syndrome). Braz J Otorhinolaryngol 77:455-460.

Salvado A, Rodriguez K, Guarisco JL. 2003. Hemifacial microsomia. J La State Med Soc 155:136-141.

Strömland K, Miller M, Sjögreen L, Johansson M, Joelsson BME, Billstedt E, et al. 2007. Oculo-auriculo-vertebral spectrum: Associated anomalies, functional deficits and possible development risk factors. Am J Med Genet Part A 143A:1317-1325.

Van Lierde KM, Van Cauwenberge P, Stevens I, Dhooge I. 2004. Language, articulation, voice and resonance characteristics in 4 children with Goldenhar syndrome: A pilot study. Folia Phoniatr Logop 56:131-143.

Vinay C, Reddy RS, Uloopi KS, Madhuri V, Sekhar RC. 2009. Craniofacial features in Goldenhar syndrome. J Indian Soc Pedod Prev Dent 27:121-124.

Werler MM, Sheehan JE, Hayes C, Padwa BL, Mitchell AA, Mulliken JB. 2004. Demografic and reproductive factors associated with hemifacial microsomia. Cleft Palate Craniofac J 41:494-500.

Yule W, Rutter M. 1987. Language development and disorders. Oxford: MacKeith Press/Blackwell. 